\title{
PLCE1 Polymorphisms and Risk of Esophageal and Gastric Cancer in a Northwestern Chinese Population
}

\author{
Ping Liang, ${ }^{1}$ Wentao Zhang, ${ }^{1}$ Weihua Wang, ${ }^{1}$ Peng Dai, ${ }^{1}$ Qin Wang, ${ }^{1}$ Wei Yan, ${ }^{2}$ Wei Wang, \\ Xiaoying Lei, ${ }^{1}$ Daxiang Cui $\left(\mathbb{D},{ }^{3}\right.$ and Zhen Yan $(\mathbb{D})^{1}$ \\ ${ }^{1}$ State Key Laboratory of Cancer Biology, Department of Pharmacogenomics, School of Pharmacy, \\ The Fourth Military Medical University, Xian 710032, China \\ ${ }^{2}$ Department of Pathology, Xijing Hospital, The Fourth Military Medical University, Xian 710032, China \\ ${ }^{3}$ Institute of Nano Biomedicine and Engineering, Key Laboratory for Thin Film and Microfabrication of Ministry of Education, \\ Research Institute of Translation Medicine, Shanghai Jiao Tong University, Shanghai 200240, China
}

Correspondence should be addressed to Daxiang Cui; dxcui@sjtu.edu.cn and Zhen Yan; yanzhen@fmmu.edu.cn

Received 21 September 2018; Revised 7 January 2019; Accepted 22 January 2019; Published 25 February 2019

Academic Editor: Hon W. Koon

Copyright (C) 2019 Ping Liang et al. This is an open access article distributed under the Creative Commons Attribution License, which permits unrestricted use, distribution, and reproduction in any medium, provided the original work is properly cited.

\begin{abstract}
The reported risk susceptibility between phospholipase C epsilon 1 (PLCE1) polymorphisms and esophageal cancer (EC) and gastric cancer (GC) remained inconsistent and controversial, especially on variants other than rs2274223. The relationship between PLCE1 polymorphisms and gene expression is also unclear. Here we conducted a case-control study from northwest China, genotyped seven tag single nucleotide polymorphisms (SNPs) in PLCE1 with multiplexed SNP MassARRAY assay. Stratified analysis was carried out and PLCE1 expression was evaluated in specified groups with the method of qRT-PCR and immunohistochemistry. Results showed that the minor alleles of rs3765524, rs2274223, and rs10509670 were associated with increased risk of EC and GC. Linkage disequilibrium analysis revealed protective haplotypes of CCAAGTC and CCAA. By stratification, a more significant association was found in subgroups of male, age $\geq 54$, tumor stages of I-II and tumor size $\leq 5 \mathrm{~cm}$, EC and cardia cancer (CC) of stomach, and moderate to well differentiated squamous carcinoma. In addition, a significant association for rs3765524 with noncardia cancer (NCC) and adenocarcinoma which is predominant in China was also observed. Further expression analysis identified that PLCE1 was downregulated in NCC tissues comparing to their adjacent noncancerous tissues, and its protein expression was higher in genotype rs3765524 CT/TT than in rs3765524 CC. In summary, our study suggests that PLCE1 polymorphisms may affect its gene expression and are associated with not only EC and CC, but also, to some extent, NCC risk in this study population.
\end{abstract}

\section{Introduction}

Esophageal cancer (EC) and gastric cancer (GC) are the two most common cancers originating from digestive tract around world [1], especially in China [2,3]. There are many differences between EC and GC, such as genetic background, histological type, and Helicobacter pylori infection, while they are both known to be the results of complex interactions between inherited and environmental factors $[4,5]$.

Phospholipase C epsilon 1 (PLCE1) gene was reported to locate at $10 \mathrm{q} 23$, encoding a member of the human phosphoinositide-specific phospholipase C family [6]. It has been involved in the regulation of cell growth, differentiation, and oncogenesis [7]. Genome wide association studies (GWAS) have identified single nucleotide polymorphisms (SNPs), mostly rs2274223 A $>\mathrm{G}$, and rs3765524 C $>\mathrm{T}$ in PLCE1 gene as shared susceptibility loci for EC and GC [8-10].

Although several independent candidate-gene studies have confirmed the association between EC, GC, and PLCE1 SNPs subsequently, there is more limited data on variants other than rs2274223, especially for GC [11-24]. Moreover, whether these loci are associated with noncardia cancer in addition to cardia and esophageal is not clear; whether or not PLCE1 polymorphisms affect gene expression and protein 
function were only reported in few contradictory results [9, $14,15,23,25-28]$.

To further explore the association between PLCE1 polymorphisms and risk of EC and GC or their subtypes, we collected blood samples from Chinese northwestern population and used multiplexed SNP MassARRAY assay to sequence a panel of tag SNPs (tSNPs) of PLCE1 in a case-control study. We completed a comprehensive analysis by logistic regression and stratification method and examined the expression of PLCE1 in tissue samples.

\section{Material and Methods}

2.1. Study Population. A total of 324 GC or EC patients and 357 control volunteer individuals without known malignancies in the Xijing hospital of the Fourth Military Medical University in Xian city, China, during 2009 to 2012 were enrolled in the study. The cases had no previous history of other cancers, or prior chemotherapy or radiotherapy. All of the chosen subjects were Chinese Han living in Xi'an city and its surrounding areas. Generally, subjects with chronic diseases and conditions involving vital organs (heart, lung, liver, kidney, and brain) and severe endocrinological, metabolic, and nutritional diseases were excluded from this study. The purpose of the above exclusion procedures was to minimize the known environmental and therapeutic factors that influence the variation of human complex diseases. Peripheral blood samples from GC and EC patients were collected before or after surgery. Formalin-fixed, paraffinembedded cancer and paired adjacent noncancerous tissues were collected after surgery from part of the GC patients. Patients' clinical data and postoperative pathological reports including the pathological types, pTNM and clinical stages, and the degrees of tumor differentiation were indexed from medical records. The study was approved by the Ethical Committee of Xijing Hospital (Xi'an, China), and this study complied with the World Medical Association Declaration of Helsinki. Informed consent was given by all the subjects for participation in this study.

2.2. DNA Isolation and Genotyping Assays. A panel of seven tSNPs of rs3765524, rs3818432, rs2274223, rs10509670, rs11187852, rs3781264, and rs11187866 in PLCE1 gene were included in this study. All the SNPs have a disequilibrium $\left(\mathrm{D}^{\prime}\right)$ threshold of 0.8 and minor allele frequency (MAF) $>0.05$ in the HapMap Chinese Han population. Genomic DNA was extracted from peripheral blood using a Blood DNA Extraction Kit (TIANGEN, China), quantified with NanoDrop 2000 (Thermo, USA) and stored at $-20^{\circ} \mathrm{C}$ until use. Primers were designed in a multiplexed SNP MassEXTEND assay with the Sequenom MassARRAY Assay Design 3.0 Software. SNP genotyping was performed by Sequenom MassARRAY RS1000 as reported previously [29]. Data management was conducted and analyzed by Sequenom Typer 4.0 Software.

2.3. Quantitative Real-Time PCR. Total RNA was extracted from tissue samples with E.Z.N.A.TM FFPE RNA Kit (OMEGA, USA). The protocol of total RNA isolation, cDNA preparation, and qRT-PCR was as reported previously by using the PrimeScriptTM RT Master Mix (Takara, Japan) on a 7500 fast real-time PCR system (Applied Biosystems) [29]. We used the following primers covering the two PLCE1 spliceosomes, respectively: PLCE1A, forward 5'-ATCATAGAGACAGGCAGAGCACA- $3^{\prime}$ and reverse $5^{\prime}$-ATGCCACATAGTTTTTCTTTTGC- $3^{\prime}$; PLCE1B, forward $5^{\prime}$ GATTAATGGTTTCAGAAGGAAGTGC- $3^{\prime}$ and reverse $5^{\prime}$ CTCCAGCATCCACATCCATCC- $3^{\prime}$. Human $\beta$-actin was used as an endogenous control. For each sample, we calculated the difference in threshold cycles for each PLCE1 copy by the $2^{-\Delta C T}$ method.

2.4. Immunohistochemistry Staining. The procedure of immunochemistry staining has been described in our previous publication [29]. Paraffin-embedded tissue specimens were deparaffinized in xylene and then soaked in ethanol and then PBS. We performed antigen retrieval in $100 \mathrm{mM}$ sodium citrate buffer at $100^{\circ} \mathrm{C}$ for $20 \mathrm{~min}$. Subsequently, we blocked endogenous peroxidase activity in $3 \%$ hydrogen peroxide in methanol for $15 \mathrm{~min}$ and then blocked nonspecific binding in $5 \%$ normal goat serum overnight at $4^{\circ} \mathrm{C}$. We incubated sections for 2 hours at room temperature with rabbit antiPLCE1 (SIGMA, HPA015598, 1:20 dilution) antibody, and then with alkaline phosphatase conjugated anti-rabbit IgG antibody. We visualized PLCE1 protein by Histostain ${ }^{\mathrm{TM}}-$ Plus Kits (ZYMED, SP-9001). At least three experienced pathologists examined the staining using the following criteria: strong positive (signal in the cancer cells is stronger than the normal gastric gland), positive (signal in the cancer cells is as strong as that in a normal gastric gland), weak positive (signal between positive and negative), and negative (signal is no more than the background signal in the surrounding stromal cells).

2.5. Statistical Analysis. We performed statistical analysis using Microsoft Excel and SPSS 16.0 statistical package (SPSS, Chicago, IL). All $P$ values in this study were two-sided. We considered $P \leq 0.05$ the threshold for statistical significance. We tested genotypic frequencies in control subjects for each SNP for departure from HWE using an exact test. We compared genotype frequencies of case and control subjects using the $\mathrm{Chi}^{2}$ test. We calculated OR and $95 \% \mathrm{CI}$ by unconditional logistic regression analysis. There were two factors of age and gender adjusted for the analysis. We used the Haploview program to estimate the pairwise LD between markers and partition haplotype blocks. We inferred haplotypes using the Haploview software package (version 4.2).

\section{Results}

3.1. Overall Association between the PLCE1 tSNPs and the Risk of EC and GC. The characteristics of all cases and controls included in the study were listed in Table 1. Seven tSNPs in PLCE1 gene were genotyped and all of the tSNPs were in Hardy-Weinberg equilibrium (HWE) in the control population $(P>0.05)$. 
TABLE 1: Characteristics of case and control.

\begin{tabular}{|c|c|c|c|c|}
\hline \multirow{3}{*}{$\begin{array}{l}\text { Variables } \\
\text { Age, mean years (SD) }\end{array}$} & \multirow{2}{*}{\multicolumn{2}{|c|}{$\begin{array}{c}\text { Case (324) } \\
\text { No. (\%) }\end{array}$}} & \multirow{2}{*}{\multicolumn{2}{|c|}{$\begin{array}{c}\text { Control (357) } \\
\text { No. (\%) }\end{array}$}} \\
\hline & & & & \\
\hline & 57.4 & $(11.4)$ & 50.4 & $(12.7)$ \\
\hline \multicolumn{5}{|l|}{ Sex } \\
\hline Male & 252 & $(77.8)$ & 212 & $(59.4)$ \\
\hline Female & 72 & $(22.2)$ & 145 & $(40.6)$ \\
\hline \multicolumn{5}{|l|}{ Tumor site } \\
\hline EC & 48 & $(14.8)$ & & \\
\hline $\mathrm{CC}$ & 35 & $(10.8)$ & & \\
\hline NCC & 241 & $(74.4)$ & & \\
\hline \multicolumn{5}{|l|}{ Pathology } \\
\hline Adenocarcinoma & 223 & $(68.8)$ & & \\
\hline Squamous carcinoma & 38 & $(11.7)$ & & \\
\hline Unspecified & 63 & $(19.4)$ & & \\
\hline \multicolumn{5}{|l|}{ Differentiation } \\
\hline Poor & 117 & $(36.1)$ & & \\
\hline Moderate to well & 117 & $(36.1)$ & & \\
\hline Unspecified & 90 & $(27.8)$ & & \\
\hline \multicolumn{5}{|l|}{ Tumor size } \\
\hline$\leq 5 \mathrm{~cm}$ & 153 & $(36.2)$ & & \\
\hline$>5 \mathrm{~cm}$ & 71 & $(16.8)$ & & \\
\hline Unspecified & 199 & $(47.0)$ & & \\
\hline \multicolumn{5}{|l|}{ Tumor stage } \\
\hline I-II & 174 & $(53.7)$ & & \\
\hline III-IV & 62 & $(19.1)$ & & \\
\hline Unspecified & 88 & $(27.2)$ & & \\
\hline
\end{tabular}

EC, esophageal cancer; CC, cardia cancer; NCC, noncardia cancer.

After genotyping, we conducted logistic regression to evaluate the association of each tSNP with the risk of EC and GC (Table 2). Results showed that there were three tSNPs (rs3765524, rs2274223, and rs10509670) associated with the risk of EC and GC: rs3765524 (CT vs CC, OR $=1.66,95 \% \mathrm{CI}$ $1.16-2.38, P=0.006$; $\mathrm{CT} / \mathrm{TT}$ vs $\mathrm{CC}, \mathrm{OR}=1.65,95 \% \mathrm{CI} 1.17$ 2.34, $P=0.004$ ); rs 2274223 (AG vs AA, $\mathrm{OR}=1.57,95 \% \mathrm{CI}$ $1.10-2.26, P=0.014$; AG/GG vs AA, OR $=1.55,95 \% \mathrm{CI} 1.10$ 2.20, $P=0.013$ ); rs10509670 (AG vs AA, OR $=1.54,95 \% \mathrm{CI}$ $1.07-2.21, P=0.019$; AG/GG vs AA, $\mathrm{OR}=1.54,95 \%$ CI 1.09 $2.18, P=0.014)$.

3.2. Linkage Disequilibrium and Haplotype Evaluation for the PLCE1 tSNPs. Linkage disequilibrium (LD) analysis revealed that the seven tSNPs of PLCE1 linked with each other (Figure 1). Haplotype "CCAAGTC" accounted for $71.5 \%$ of the whole haplotypes in EC and GC cases. This is a protective haplotype against the risk of EC/GC (OR $=0.72 ; 95 \% \mathrm{CI}$ $=0.53-0.97 ; P=0.029)$ (Table 3). Further analysis revealed that the LD block could be divided into two subblocks (Figure 1(a)). Subblock $1\left(\mathrm{r}^{2}>0.79\right)$ was composed of four tSNPs of rs3765524, rs3818432, rs2274223, and rs10509670, where the three risk SNPs identified above were included. Subblock $2\left(\mathrm{r}^{2}>0.87\right)$ included the later three tSNPs of rs11187852, rs3781264, and rs11187866. In subblock 1, "CCAA" accounted for $72.4 \%$ of the whole haplotypes in EC/GC cases and was found to be the protective haplotype against the risk of $\mathrm{EC} / \mathrm{GC}(\mathrm{OR}=0.67 ; 95 \% \mathrm{CI}=0.49-0.91 ; P=$ $0.009)$.

3.3. Stratified Analysis for the Clinicopathologic Data of Patients. Anatomically, gastric cancer includes cardia cancer (CC) and noncardia cancers (NCC). Pathologically, gastric cancer has adenocarcinoma and squamous carcinoma. Then we performed a stratified analysis to determine the association between the three tSNPs (rs3765524, rs2274223, and rs10509670) and clinicopathologic data in dominant model (Table 4). Significant association between the three tSNPs and risk of EC and GC was observed for subgroup patients of male, age $\geq 54$, tumor stages of I-II and tumor size $\leq$ $5 \mathrm{~cm}$, EC and cardia cancer (CC), and moderate to well differentiated squamous carcinoma. In addition, a significant association for rs3765524 with noncardia cancer (NCC) and adenocarcinoma was also observed.

3.4. Expression Distribution of PLCE1 Protein in Stomach Tissue. Now that the association between PLCE1 polymorphisms and GC risk exhibited disparity according to the tumor subsites, we then evaluated the expression distribution of PLCE1 protein in human GC and adjacent noncancer tissues (ANC) by tissue microarray. In the ANC tissue, 
TABLE 2: Logistic regression of candidate tSNPs in PLCE1 and their association with EC and GC risk.

\begin{tabular}{|c|c|c|c|c|c|c|c|c|c|c|c|c|}
\hline \multirow{2}{*}{$\begin{array}{l}\text { ID } \\
\text { rs3765524 }\end{array}$} & \multirow{2}{*}{$\frac{\text { Genotype }}{\text { CC }}$} & \multicolumn{2}{|c|}{$\begin{array}{c}\text { Case }(\mathrm{n}=324) \\
\text { No. }(\%)\end{array}$} & \multicolumn{2}{|c|}{$\begin{array}{c}\text { Control }(\mathrm{n}=357) \\
\text { No. }(\%)\end{array}$} & \multicolumn{2}{|c|}{$\begin{array}{c}\text { Crude OR } \\
(95 \% \mathrm{CI})\end{array}$} & \multirow[t]{2}{*}{$P$} & \multicolumn{2}{|c|}{$\begin{array}{l}\text { Adjusted OR } \\
(95 \% \mathrm{CI})\end{array}$} & \multirow[t]{2}{*}{$P$} & \multirow[t]{2}{*}{ HWE-P } \\
\hline & & 168 & $(51.9)$ & 223 & $(62.6)$ & 1 & & & 1 & & & \\
\hline & CT & 136 & $(42.0)$ & 117 & (32.9) & 1.54 & $(1.12-2.12)$ & 0.008 & 1.66 & $(1.16-2.38)$ & 0.006 & \multirow{3}{*}{0.896} \\
\hline & TT & 20 & $(6.2)$ & 16 & $(4.5)$ & 1.66 & $(0.83-3.30)$ & 0.149 & 1.60 & $(0.72-3.55)$ & 0.247 & \\
\hline & $\mathrm{CT} / \mathrm{TT}$ & 156 & $(48.1)$ & 133 & $(37.4)$ & 1.56 & $(1.15-2.11)$ & 0.005 & 1.65 & $(1.17-2.34)$ & 0.004 & \\
\hline \multirow[t]{4}{*}{ rs3818432 } & CC & 104 & $(57.5)$ & 193 & $(65.6)$ & 1 & & & 1 & & & \multirow{4}{*}{0.856} \\
\hline & $\mathrm{CA}$ & 73 & $(40.3)$ & 91 & $(31.0)$ & 1.49 & $(1.01-2.20)$ & 0.045 & 1.47 & $(0.97-2.23)$ & 0.073 & \\
\hline & $\mathrm{AA}$ & 4 & $(2.2)$ & 10 & $(3.4)$ & 0.74 & $(0.23-2.42)$ & 0.622 & 0.87 & $(0.24-3.09)$ & 0.826 & \\
\hline & CA/AA & 77 & $(42.5)$ & 101 & $(34.4)$ & 1.41 & $(0.97-2.07)$ & 0.074 & 1.41 & $(0.94-2.13)$ & 0.098 & \\
\hline \multirow[t]{4}{*}{ rs2274223 } & $\mathrm{AA}$ & 177 & $(54.8)$ & 229 & $(64.1)$ & 1 & & & 1 & & & \multirow{4}{*}{0.457} \\
\hline & AG & 126 & $(39.0)$ & 111 & $(31.1)$ & 1.47 & $(1.06-2.03)$ & 0.019 & 1.57 & $(1.10-2.26)$ & 0.014 & \\
\hline & GG & 20 & $(6.2)$ & 17 & $(4.8)$ & 1.52 & $(0.77-2.99)$ & 0.223 & 1.43 & $(0.66-3.14)$ & 0.367 & \\
\hline & AG/GG & 146 & $(45.2)$ & 128 & (35.9) & 1.48 & $(1.08-2.01)$ & 0.013 & 1.55 & $(1.10-2.20)$ & 0.013 & \\
\hline \multirow[t]{4}{*}{ rs10509670 } & $\mathrm{AA}$ & 178 & (54.9) & 229 & $(64.5)$ & 1 & & & 1 & & & \multirow{4}{*}{0.391} \\
\hline & AG & 124 & $(38.3)$ & 109 & $(30.7)$ & 1.46 & $(1.06-2.02)$ & 0.021 & 1.54 & $(1.07-2.21)$ & 0.019 & \\
\hline & GG & 22 & $(6.8)$ & 17 & $(4.8)$ & 1.66 & $(0.86-3.23)$ & 0.132 & 1.54 & $(0.72-3.32)$ & 0.268 & \\
\hline & AG/GG & 146 & $(45.1)$ & 126 & $(35.5)$ & 1.49 & $(1.10-2.03)$ & 0.011 & 1.54 & (1.09-2.18) & 0.014 & \\
\hline \multirow[t]{4}{*}{ rs11187852 } & GG & 122 & $(67.4)$ & 212 & $(72.1)$ & 1 & & & 1 & & & \multirow{4}{*}{0.506} \\
\hline & GA & 56 & $(30.9)$ & 77 & $(26.2)$ & 1.26 & $(0.84-1.90)$ & 0.263 & 1.33 & $(0.85-2.07)$ & 0.212 & \\
\hline & $\mathrm{AA}$ & 3 & $(1.7)$ & 5 & $(1.7)$ & 1.04 & $(0.24-4.44)$ & 0.955 & 1.68 & $(0.34-8.18)$ & 0.522 & \\
\hline & GA/AA & 59 & $(32.6)$ & 82 & $(27.9)$ & 1.25 & $(0.84-1.87)$ & 0.276 & 1.34 & $(0.87-2.08)$ & 0.185 & \\
\hline \multirow[t]{4}{*}{ rs3781264 } & $\mathrm{TT}$ & 120 & $(66.3)$ & 206 & $(70.1)$ & 1 & & & 1 & & & \multirow{4}{*}{0.511} \\
\hline & $\mathrm{TC}$ & 58 & $(32.0)$ & 82 & $(27.9)$ & 1.21 & $(0.81-1.82)$ & 0.347 & 1.24 & $(0.80-1.92)$ & 0.331 & \\
\hline & $\mathrm{CC}$ & 3 & $(1.7)$ & 6 & $(2.0)$ & 0.86 & $(0.21-3.49)$ & 0.831 & 1.07 & $(0.23-5.00)$ & 0.932 & \\
\hline & $\mathrm{TC} / \mathrm{CC}$ & 61 & $(33.7)$ & 88 & $(29.9)$ & 1.19 & $(0.80-1.77)$ & 0.390 & 1.23 & $(0.80-1.89)$ & 0.340 & \\
\hline \multirow[t]{4}{*}{ rs11187866 } & $\mathrm{CC}$ & 120 & $(66.7)$ & 210 & $(71.4)$ & 1 & & & 1 & & & \multirow{4}{*}{0.723} \\
\hline & CG & 56 & $(31.1)$ & 76 & (25.9) & 1.29 & $(0.85-1.95)$ & 0.226 & 1.33 & $(0.85-2.08)$ & 0.210 & \\
\hline & GG & 4 & $(2.2)$ & 8 & $(2.7)$ & 0.88 & $(0.26-2.97)$ & 0.830 & 1.13 & $(0.30-4.23)$ & 0.857 & \\
\hline & $\mathrm{CG} / \mathrm{GG}$ & 60 & $(33.3)$ & 84 & $(28.6)$ & 1.25 & $(0.84-1.86)$ & 0.274 & 1.31 & $(0.85-2.03)$ & 0.218 & \\
\hline
\end{tabular}

Notes. The adjusted OR is derived from the correction for age and gender. The results were in bold, if the $P$ value $<0.05$.

EC, esophageal cancer; GC, gastric cancer.

TABLE 3: Haplotypes of PLCE1 and their association with EC and GC risk.

\begin{tabular}{|c|c|c|c|c|c|c|c|c|}
\hline \multirow{2}{*}{ ID } & \multirow{2}{*}{ Block } & \multirow{2}{*}{ Haplotype } & \multicolumn{2}{|c|}{ Frequency $^{\mathrm{a}}$} & \multirow{2}{*}{ OR } & \multirow{2}{*}{$(95 \% \mathrm{CI})$} & \multirow{2}{*}{$\mathrm{Chi}^{2}$} & \multirow{2}{*}{$P^{\mathrm{b}}$} \\
\hline & & & Case & Control & & & & \\
\hline 1 & \multirow[t]{5}{*}{ Global } & CCAAGTC & $71.5 \%$ & $77.8 \%$ & 0.72 & $(0.53-0.97)$ & 4.742 & 0.029 \\
\hline 2 & & TAGGACG & $16.8 \%$ & $14.6 \%$ & 1.18 & $(0.83-1.69)$ & 0.861 & 0.354 \\
\hline 3 & & TAGGGTC & $4.7 \%$ & $2.9 \%$ & 1.66 & $(0.83-3.28)$ & 2.115 & 0.146 \\
\hline 4 & & TCAAGTC & $3.0 \%$ & $1.2 \%$ & 2.49 & $(0.97-6.41)$ & 3.834 & 0.050 \\
\hline 5 & & TCGGGTC & $2.2 \%$ & $1.2 \%$ & 1.82 & $(0.66-5.03)$ & 1.381 & 0.240 \\
\hline 6 & \multirow[t]{4}{*}{ Sub-1 } & CCAA & $72.4 \%$ & $79.7 \%$ & 0.67 & $(0.49-0.91)$ & 6.756 & 0.009 \\
\hline 7 & & TAGG & $22.1 \%$ & $17.6 \%$ & 1.33 & $(0.96-1.84)$ & 2.859 & 0.091 \\
\hline 8 & & TCAA & $3.0 \%$ & $1.2 \%$ & 2.48 & $(0.95-6.42)$ & 3.752 & 0.053 \\
\hline 9 & & TCGG & $2.2 \%$ & $1.2 \%$ & 1.81 & $(0.65-5.04)$ & 1.331 & 0.249 \\
\hline 10 & \multirow[t]{2}{*}{ Sub-2 } & GTC & $81.5 \%$ & $83.2 \%$ & 0.89 & $(0.63-1.25)$ & 0.436 & 0.509 \\
\hline 11 & & ACG & $17.1 \%$ & $14.8 \%$ & 1.19 & $(0.83-1.70)$ & 0.921 & 0.337 \\
\hline
\end{tabular}

Notes. (A) Only haplotypes with frequencies of $\geq 3 \%$ are shown. (B) The results were in bold, if the $P$ value $<0.05$.

EC, esophageal cancer; GC, gastric cancer. 


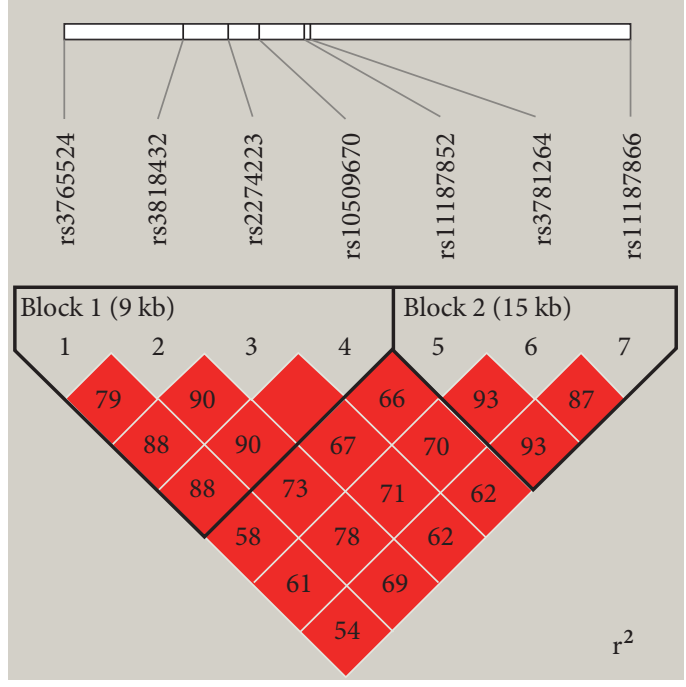

(a)

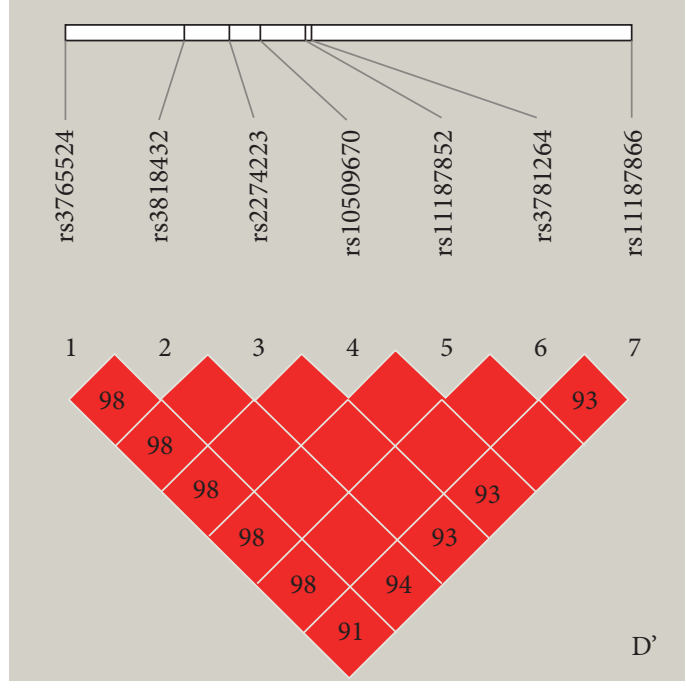

(b)

Figure 1: Linkage disequilibrium (LD) analysis of PLCE1 tSNPs. (a) $\mathrm{r}^{2}$ of LD analysis, which showed that the seven tSNPs are linked to each other and could be divided into two subblocks. (b) D' of LD analysis.

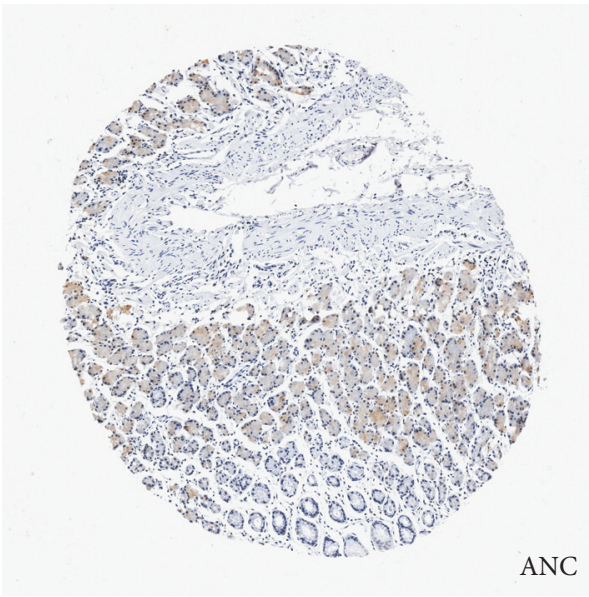

(a)

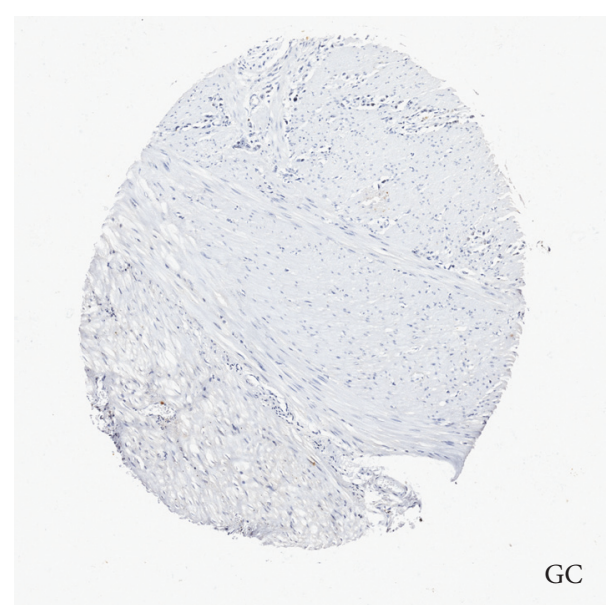

(b)

FIGURE 2: Representative images of PLCE1 expression distribution in gastric cancer (GC) and adjacent noncancerous (ANC) tissues by tissue microarray. (a) ANC tissues. PLCE1 protein was expressed in the cytoplasm of columnar epithelial cells and mainly distributed in the junction of cardia and gastric fundus glands (200x). (b) GC tissues. Structure distortion and confusion of tubular glands, heterogeneity of epithelial cells with irregular nuclear staining, and lower expression of PLCE1 were observed (200×).

PLCE1 protein expression was positive in the cytoplasm of columnar epithelial cells and mainly distributed in the junction of cardia and gastric fundus glands (Figure 2(a)). In the GC tissue, the structure distortion and confusion of tubular glands, obvious heterogeneity of epithelial cells with irregular nuclear staining, and lower expression of PLCE1 protein were observed (Figure 2(b)). These suggested, together with the results of rs3765524 genotyping by stratified analysis, that PLCE1 protein may be involved in carcinogenesis of NCC and adenocarcinoma, although more significant association has been found with EC, CC, and squamous carcinoma.
3.5. Effect of PLCE1 Polymorphisms on Its Expression in NCC and ANC Tissues. Because NCC and adenocarcinoma have been the predominant subtype of gastric cancer in China, we then examined the expression of PLCE1 in adenocarcinoma of NCC and their ANC tissues with different genotypes of rs3765524. For mRNA transcription, we identified two PLCE1 processing units, PLCE1A and PLCE1B, by quantitative realtime PCR (qRT-PCR). As presented in Figures 3(a)(i) and 3(a)(ii), compared with ANC tissues, the two procession units were both downregulated in NCC tissues (PLCE1A: 22 of 28, $78.57 \%, P=0.034$; PLCE1B: 21 of $28,75.00 \%, P=0.021$ ), while there was no significant difference between genotype 


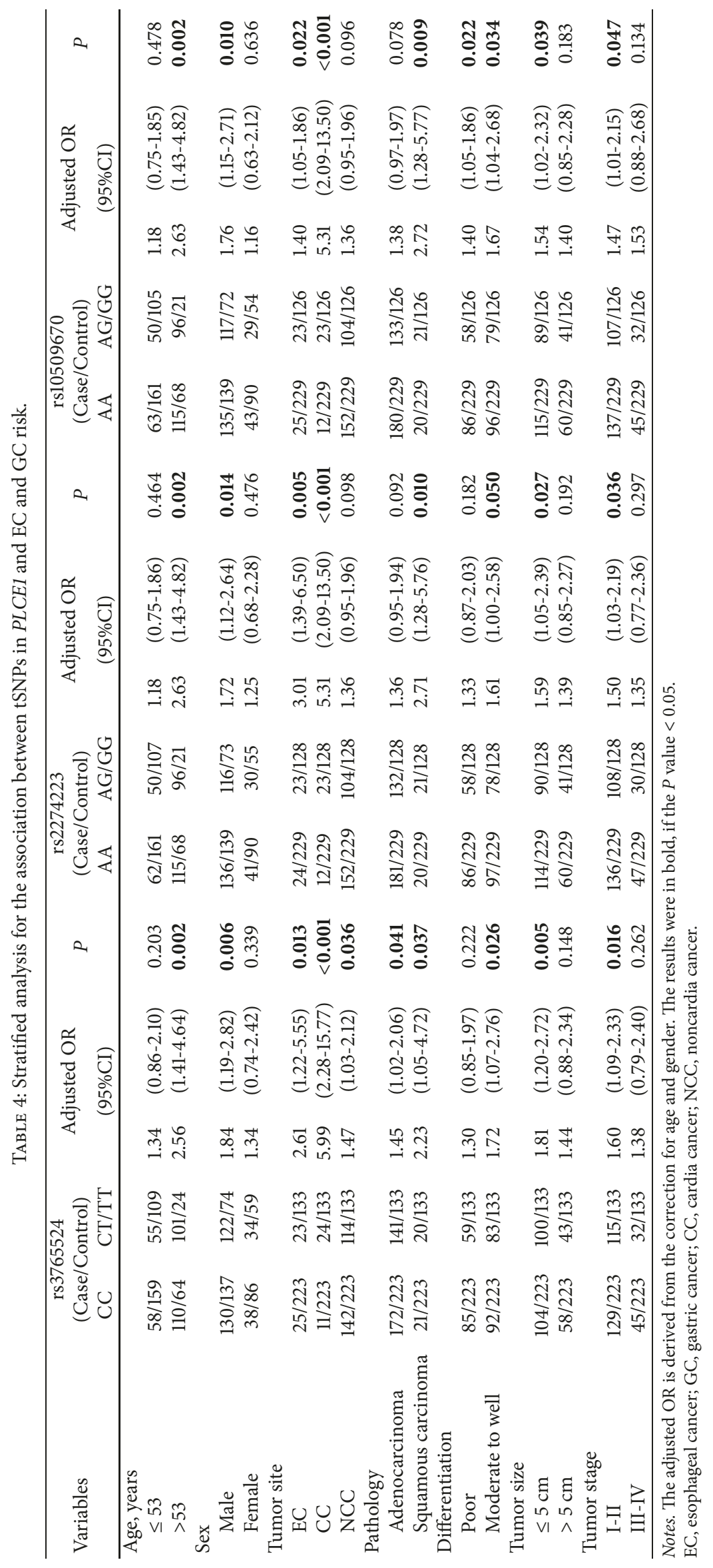




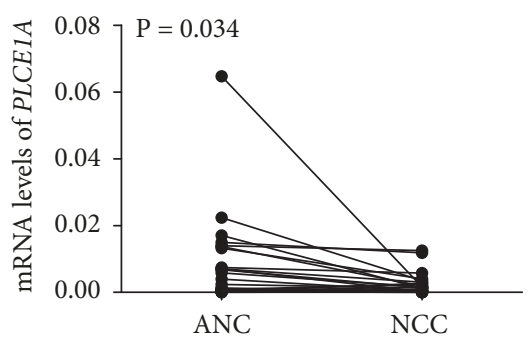

(i)

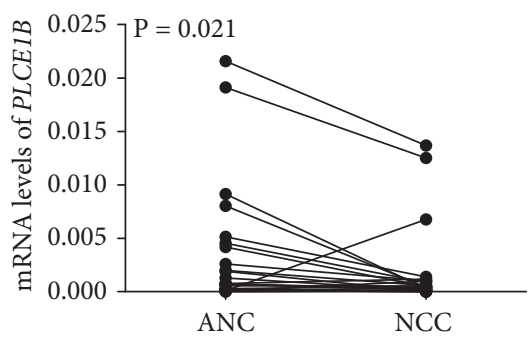

(ii)

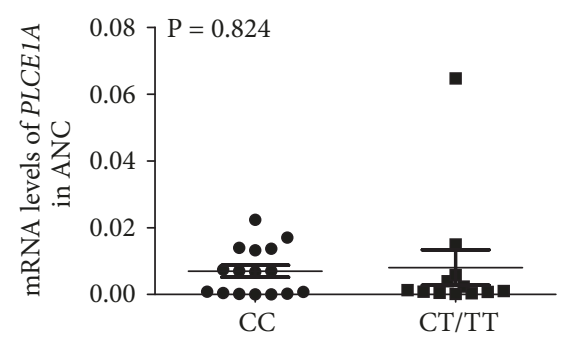

(i)

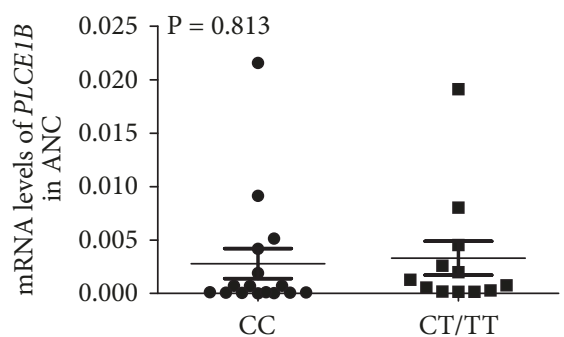

(ii)

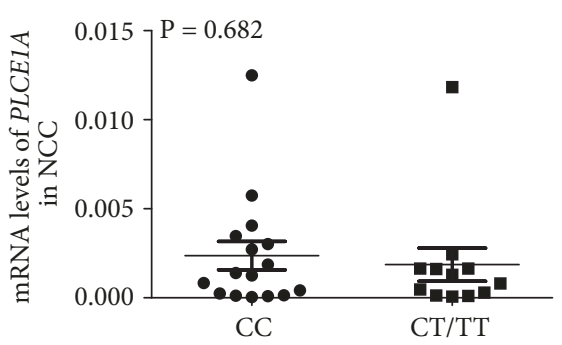

(i)

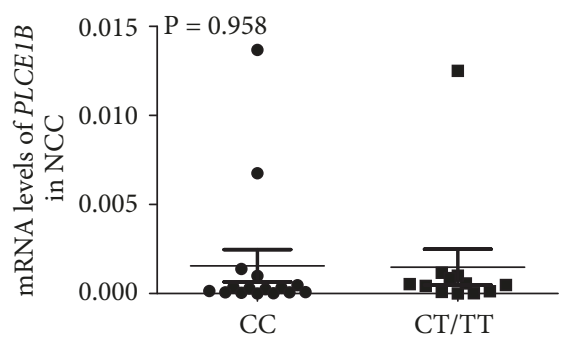

(ii)

(a)

(b)

(c)

FIGURE 3: PLCE1 mRNA expression in adenocarcinoma of noncardia cancer (NCC) and adjacent noncancerous (ANC) tissues by qRT-PCR. (a) PLCE1A (i) and PLCE1B (ii) mRNA expression in ANC and NCC tissues, respectively. (b) PLCE1A (i) and PLCE1B (ii) mRNA expression in ANC tissues with different genotypes of rs3765524. (c) PLCE1A (i) and PLCE1B (ii) mRNA expression in NCC tissues with different genotypes of rs3765524. Horizontal lines indicate mean \pm SE $(n=28)$.

rs3765524 CC and rs3765524 CT/TT both in ANC (Figures 3(b)(i) and 3(b)(ii)) and in NCC (Figures 3(c)(i) and 3(c)(ii)).

For protein translation, immunohistochemistry (IHC) staining revealed that PLCE1 protein expression was generally downregulated in NCC than in their ANC tissues regardless of rs3765524 genotype ( 6 of 13, 46.15\%, $P=0.018$, see in Figures 4(a), 4(b), and 4(c)(i)). By genotyping, the PLCE1 protein expression was found higher in group of rs3765524 CT/TT than in group of rs3765524 CC both in ANC (Figure 4(c)(ii). $P=0.031$ ) and NCC tissues (Figure 4(c)(iii). $P=0.045$ ).

\section{Discussion}

SNPs are the most common type of genetic variation, which makes them excellent biological markers [30]. On the other hand, SNPs, including those that fall within the coding or noncoding regions of genes, may affect the gene transcription and translation, as well as the structure and function of protein, contributing to changing the host susceptibility to diseases [31].

GWAS study found that some SNPs in PLCE1 corresponding to $\mathrm{Y}, \mathrm{C} 2$, and $\mathrm{RA}$ domain were associated with the risk of EC and GC [8-10]. These are very important domains to PLCE1. The Y domain folds to form the catalytic core of the phospholipase and the $\mathrm{C} 2$ domain can bind to phospholipid [32]. RA domain is in the C terminal of PLCE1 protein, which interacts directly with upstream regulators of Ras, Rap, and others [33]. The genomic region for Y, C2, and RA domains spans from exon 24 to exon 33. By referring to the frequencies of SNPs in Chinese Han population in HapMap database, after removing the SNPs with minimum allele frequency (MAF) less than 0.05, seven candidate SNPs in the region were selected in our study, where rs3765524 was in exon 24 and in Y domain, rs3818432 was in intron 24, rs2274223 was in exon 26 and in C2 domain, rs10509670 was in intron 26, rs11187852 and rs3781264 were in intron 27, and rs11187866 was in intron 32 .

By genotyping and logistic regression, we not only confirmed the two previous reported SNPs of rs3765524 and rs2274223 [8-10] but also revealed that another SNP of rs10509670 in PLCE1 was associated with the risk of EC and GC susceptibility. rs3765524 C $>$ T causes an amino acid change from Thr to Ile (ACC1777ATC), and rs2274223 A $>\mathrm{G}$ can also cause a missense mutation of His to Arg (CAC1927CGC). These two SNPs are corresponding to the Y and C2 domain of PLCE1 protein, respectively. We noticed that Thr, His, and Arg are frequently modified amino acid residues in human proteins. Different posttranslational modification may alter the structure, stability, and function of PLCE1 protein [34]. In the case of rs3765524, we found that although there was no difference in mRNA transcription between wild type and mutant type (Figure 3 ), there was a difference in protein expression (Figure 4). Among them, the expression of CT/TT genotype was higher than that of CC genotype in both NCC and ANC groups, implying that the amino acid change by the polymorphism of rs3765524 might lead to different protein modifications or structural changes, ultimately affecting PLCE1 expression or stability.

The third loci of rs10509670 located in the intron of PLCE1 gene has also shown to be associated with risk of EC and GC 


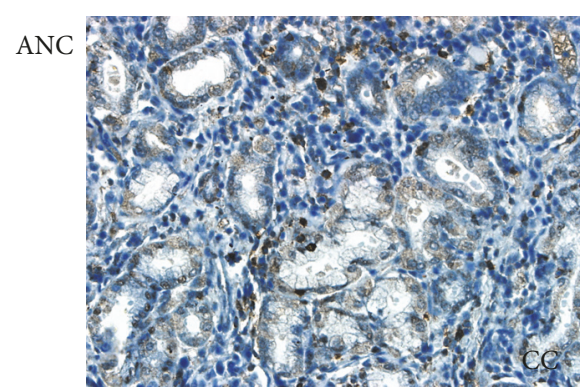

(i)

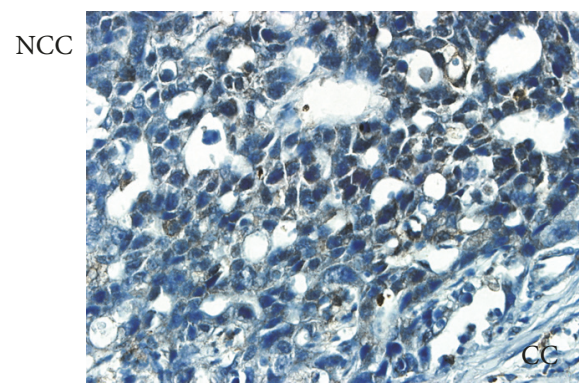

(i)

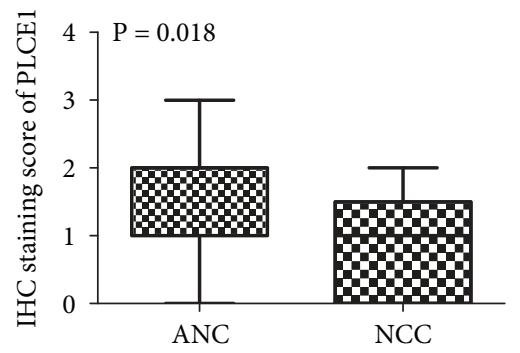

(i)

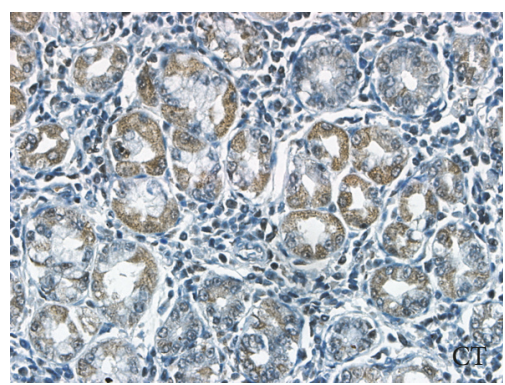

(ii)

(a)

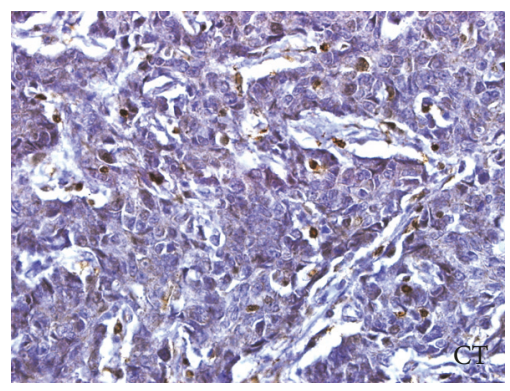

(ii)

(b)

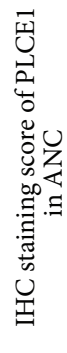

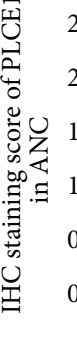

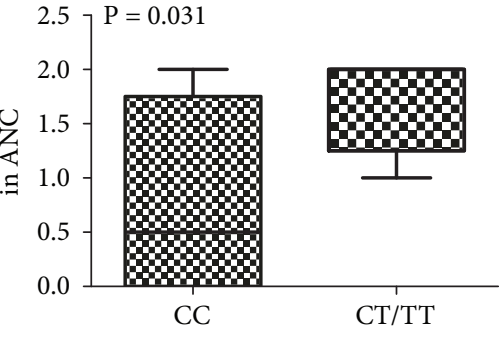

(ii)

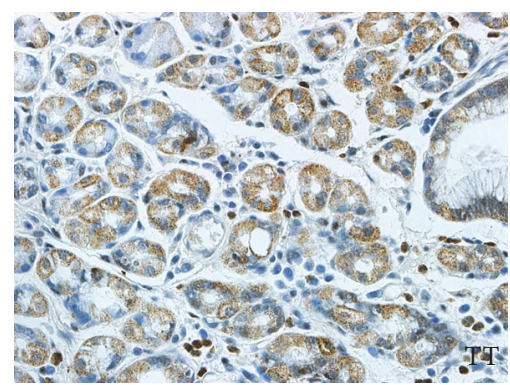

(iii)

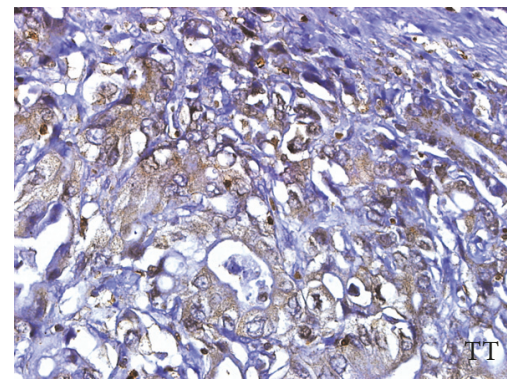

(iii)

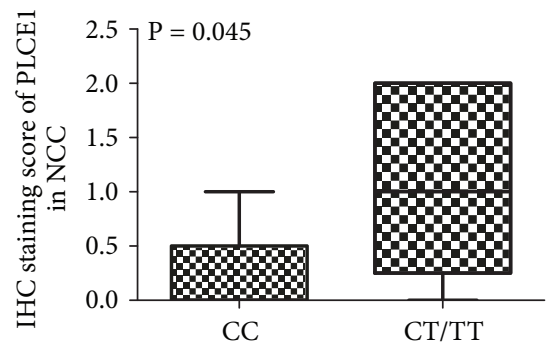

(iii)

(c)

FIGURE 4: PLCE1 protein expression in adenocarcinoma of noncardia cancer (NCC) and adjacent noncancerous (ANC) tissues by immunohistochemistry (IHC) staining. (a) Representative images of PLCE1 expression in ANC tissues with different genotypes of rs3765524 ((i) CC, (ii) CT, and (iii) TT) (400×). (b) Representative images of PLCE1 expression in NCC tissues with different genotypes of rs3765524 ((i) CC, (ii) CT, and (iii) TT) (400×). (c) IHC staining score comparison of PLCE1 (i) between ANC and NCC tissues regardless of rs3765524 genotyping, (ii) in ANC tissues with different genotypes of rs3765524, (iii) in NCC tissues with different genotypes of rs3765524. The data was represented as mean \pm SE $(n=13)$.

in the experiment. We hypothesize that rs10509670 A>G may affect PLCEl gene structure or expression by regulating gene splicing or transcription [31]. In the study, the seven tSNPs have been proved to be in LD. Moreover, we identified two haplotypes associated with EC and GC risk. The haplotype of "CCAAGTC" (corresponding to Y, C2, and RA domains) and the haplotype in subblock 1 of "CCAA" (corresponding to Y and $\mathrm{C} 2$ domains) have decreased risk of EC and GC of 33\% and $28 \%$, respectively.

Previous studies have exhibited different associations between PLCE1 polymorphisms and the risk of EC and GC, especially for different tumor subsites of GC in several candidate-gene studies [11-24]. The latest large meta-analyses confirmed the G allele of PLCE1 rs2274223 to be associated with an increased risk of cardia cancer (CC) rather than noncardia cancer (NCC) [35]. In our stratification analysis, we not only confirmed the $\mathrm{T}$ allele of rs 3765524 and $\mathrm{G}$ allele of rs2274223 but also identified that the $G$ allele of rs10509670 was associated with increased risk of EC and CC susceptibility. Furthermore, we revealed a significant association of rs $3765524 \mathrm{C}>\mathrm{T}$ with the increased risk of NCC and adenocarcinoma. As we know, NCC has predominant incidence among digestive tract tumors in China [36,37].

So far, the literature reports about PLCE1 expression and distribution were still unclear and conflicting. Previously, we conducted a comprehensive analysis of PLCE1 expression 
in atrophic gastritis and GC tissues, which revealed that differential expression of PLCE1 may distinguish GC from inflammation lesions [28]. In terms of tumorous-normal comparison, upregulation and downregulation of PLCE1 were both found in EC and CC at mRNA and/or protein levels $[9,15,25,26]$, while there was only one study that identified downregulation of PLCE1 at mRNA level in NCC [26]. In terms of the comparison of minor-major alleles of rs2274223 with PLCE1 expression, the results for EC were also inconsistent $[14,15,38]$, and there is no report about CC and NCC until now. Another two studies reported the expression of PLCE1 in GC but without specific tumor subsites information (CC or NCC), which presented opposite conclusions for tumorous to normal comparison $[23,28]$.

By tissue microarray, we identified that PLCE1 protein is expressed not only in cardia but also in gastric fundus glands both in GC and ANC tissues. This result, together with the association of rs3765524 C>T with NCC risk, suggests that PLCE1 protein may be involved in carcinogenesis of NCC. Therefore, we used qRT-PCR and IHC to study genetic variation effects on PLCE1 expression in NCC and their ANC tissues. Results showed that the expression of PLCE1 at both mRNA and protein levels was lower in NCC tissues than in their ANC tissues, which supports the hypothesis that PLCE1 may function as a tumor suppressor. We also found that rs3765524 genotype may affect PLCE1 expression, where PLCE1 expression was higher in group of rs3765524 $\mathrm{CT} / \mathrm{TT}$ than in group of CC. This strongly suggests, as one of the contributors, the reference allele C of rs3765524 loss of expression in tumor, but the mutated $\mathrm{T}$ allele, on the other hand, produces a "dominant negative" phenotype, which is related to the increased risk of NCC. Of course, the exact mechanism needs to be studied further. To our knowledge, this is the first report about PLCE1 expression distribution in NCC by genotypes.

PLCE1A and PLCE1B arise from alternative splicing at the amino terminus of PLCE1 protein. PLCE1A is composed of 2303aa. PLCE1B is composed of 1994aa which is truncated at the amino terminal of the peptide [39]. The different distribution and function of the two subunits in gastric carcinogenesis have not been studied yet. We demonstrated, through qRT-PCR, that both PLCE1A and PLCE1B were downregulated in NCC than their ANC tissues. This suggests that PLCE1A and PLCE1B may be involved in NCC carcinogenesis.

\section{Conclusion}

Our study reveals that PLCE1 polymorphisms may affect gene expression and function and are associated with the risk of not only EC and CC, but also, to some extent, NCC in northwestern Chinese population. The tSNPs of PLCE1 may have a potential possibility to be biomarkers for prewarning and diagnosis against these diseases.

\section{Data Availability}

The data used to support the findings of this study are available from the corresponding author upon request.

\section{Conflicts of Interest}

The authors declare that there are no conflicts of interest regarding the publication of this article.

\section{Authors' Contributions}

All authors contributed to the design, experiment of the study, and the preparation and critical revision of the manuscript and agreed to be accountable for all aspects of the study. The manuscript is approved by all authors for publication. Ping Liang and Wentao Zhang contributed equally to this work.

\section{Acknowledgments}

This work was supported by grants from the National Natural Science Foundation of China (81272276, 81225010, and 30901357), National Key Basic Research Program (973 Project: 2010CB933902), and State Key Laboratory of Cancer Biology (CBSKL2015Z14).

\section{References}

[1] F. Bray, J. Ferlay, I. Soerjomataram et al., "Global cancer statistics 2018: GLOBOCAN estimates of incidence and mortality worldwide for 36 cancers in 185 countries," CA: A Cancer Journal for Clinicians, vol. 68, no. 6, pp. 394-424, 2018.

[2] W. Chen, R. Zheng, P. D. Baade et al., "Cancer statistics in China, 2015," CA: A Cancer Journal for Clinicians, vol. 66, no. 2, pp. 115132,2016

[3] D.-X. Cui, L. Zhang, X.-J. Yan et al., "A microarray-based gastric carcinoma prewarning system," World Journal of Gastroenterology, vol. 11, no. 9, pp. 1273-1282, 2005.

[4] W. Y. Cheung and G. Liu, "Genetic variations in esophageal cancer risk and prognosis," Gastroenterology Clinics of North America, vol. 38, no. 1, pp. 75-91, 2009.

[5] H. H. Hartgrink, E. P. Jansen, N. C. van Grieken, and C. J. van de Velde, "Gastric cancer," The Lancet, vol. 374, no. 9688, pp. 477-490, 2009.

[6] C. Song, C. D. Hu, M. Masago et al., "Regulation of a novel human phospholipase C, PLCepsilon, through membrane targeting by Ras," The Journal of Biological Chemistry, vol. 276, pp. 2752-2757, 2001.

[7] S. Citro, S. Malik, E. A. Oestreich et al., "Phospholipase Cepsilon is a nexus for Rho and Rap-mediated G protein-coupled receptor-induced astrocyte proliferation," Proceedings of the National Acadamy of Sciences of the United States of America, vol. 104, no. 39, pp. 15543-15548, 2007.

[8] C. C. Abnet, N. D. Freedman, N. Hu et al., "A shared susceptibility locus in PLCE1 at 10q23 for gastric adenocarcinoma and esophageal squamous cell carcinoma," Nature Genetics, vol. 42, pp. 764-767, 2010.

[9] L. D. Wang, F. Y. Zhou, X. M. Li et al., "Genome-wide association study of esophageal squamous cell carcinoma in Chinese subjects identifies susceptibility loci at PLCE1 and C20orf54," Nature Genetics, vol. 42, pp. 759-763, 2010.

[10] C. Wu, Z. Hu, Z. He et al., "Genome-wide association study identifies three new susceptibility loci for esophageal squamous-cell carcinoma in Chinese populations," Nature Genetics, vol. 43, no. 7, pp. 679-684, 2011. 
[11] X. Jia, P. Liu, M. Zhang et al., "Genetic variants at 6p21, 10q23, $16 \mathrm{q} 21$ and 22q12 are associated with esophageal cancer risk in a Chinese Han population," International Journal of Clinical and Experimental Medicine, vol. 8, pp. 19381-19387, 2015.

[12] J.-M. Piao, M.-H. Shin, H. N. Kim et al., "Replication of results of genome-wide association studies on esophageal squamous cell carcinoma susceptibility loci in a Korean population," Diseases of the Esophagus, vol. 27, no. 8, pp. 798-801, 2014.

[13] F. Duan, W. Xie, L. Cui et al., "Novel functional variants locus in PLCE1 and susceptibility to esophageal squamous cell carcinoma: Based on published genome-wide association studies in a central Chinese population," Cancer Epidemiology, vol. 37, no. 5, pp. 647-652, 2013.

[14] X.-B. Cui, Y.-Z. Chen, X.-L. Pang et al., "Multiple polymorphisms within the PLCE1 are associated with esophageal cancer via promoting the gene expression in a Chinese Kazakh population," Gene, vol. 530, no. 2, pp. 315-322, 2013.

[15] H. Hu, J. Yang, Y. Sun et al., "Putatively functional PLCE1 variants and susceptibility to esophageal squamous cell carcinoma (ESCC): a case-control study in eastern Chinese populations," Annals of Surgical Oncology, vol. 19, no. 7, pp. 2403-2410, 2012.

[16] R. Zhou, Y. Li, N. Wang, B. Liu, Z. Chen, and L. Zuo, "PLC- $\varepsilon 1$ gene polymorphisms significantly enhance the risk of esophageal squamous cell carcinoma in individuals with a family history of upper gastrointestinal cancers," Archives of Medical Research, vol. 43, no. 7, pp. 578-584, 2012.

[17] H. Gu, G. Ding, W. Zhang et al., "Replication study of PLCE1 and C20orf54 polymorphism and risk of esophageal cancer in a Chinese population," Molecular Biology Reports, vol. 39, no. 9, pp. 9105-9111, 2012.

[18] J. Yuan, Y. Li, T. Tian et al., "Risk prediction for early-onset gastric carcinoma: a case-control study of polygenic gastric cancer in Han Chinese with hereditary background," Oncotarget , vol. 7, no. 23, pp. 33608-33615, 2016.

[19] H. Sun, X. Wu, F. Wu et al., "Associations of genetic variants in the PSCA, MUC1 and PLCE1 genes with stomach cancer susceptibility in a Chinese population," PLoS ONE, vol. 10, no. 2, Article ID e0117576, 2015.

[20] X. Mou, T. Li, J. Wang et al., "Genetic variation of BCL2 (rs2279115), NEIL2 (rs804270), LTA (rs909253), PSCA (rs2294008) and PLCE1 (rs3765524, rs10509670) genes and their correlation to gastric cancer risk based on universal tagged arrays and $\mathrm{Fe} 3 \mathrm{O} 4$ magnetic nanoparticles," Journal of Biomedical Nanotechnology, vol. 11, no. 11, pp. 2057-2066, 2015.

[21] Y. He, C. Wang, Z. Wang, and Z. Zhou, "Genetic variant PLCE1 rs2274223 and gastric cancer: more to be explored?" Gut, vol. 65, no. 2, pp. 359-360, 2016.

[22] M. Li, L. Huang, H. Qiu et al., "Helicobacter pylori infection synergizes with three inflammation-related genetic variants in the GWASs to increase risk of gastric cancer in a Chinese population," PLoS ONE, vol. 8, no. 9, Article ID e74976, 2013.

[23] M. Wang, R. Zhang, J. He et al., "Potentially functional variants of PLCE1 identified by GWASs contribute to gastric adenocarcinoma susceptibility in an Eastern Chinese population," PLoS ONE, vol. 7, no. 3, Article ID e31932, 2012.

[24] H. Zhang, G. Jin, H. Li et al., "Genetic variants at 1q22 and 10q23 reproducibly associated with gastric cancer susceptibility in a Chinese population," Carcinogenesis, vol. 32, no. 6, pp. 848-852, 2011.

[25] X.-B. Cui, X.-L. Pang, S. Li et al., "Elevated expression patterns and tight correlation of the PLCE1 and NF- $\kappa \mathrm{B}$ signaling in
Kazakh patients with esophageal carcinoma," Medical Oncology, vol. 31, no. 1, article 791, 2014.

[26] W. Li, N. Hu, V. H. Burton et al., "PLCE1 mRNA and protein expression and survival of patients with esophageal squamous cell carcinoma and gastric adenocarcinoma," Cancer Epidemiology Biomarkers \& Prevention, vol. 23, no. 8, pp. 1579-1588, 2014.

[27] Y.-Z. Chen, X.-B. Cui, J.-M. Hu et al., “Overexpression of PLCE1 in Kazakh esophageal squamous cell carcinoma: Implications in cancer metastasis and aggressiveness," APMIS-Acta Pathologica, Microbiologica et Immunologica Scandinavica, vol. 121, no. 10, pp. 908-918, 2013.

[28] J. Chen, W. Wang, T. Zhang et al., "Differential expression of phospholipase $\mathrm{C}$ epsilon 1 is associated with chronic atrophic gastritis and gastric cancer," PLOS ONE, vol. 7, no. 10, Article ID e47563, 2012.

[29] W. Zhang, P. Liang, W. Wang et al., "The influence of PSCA gene variation on its expression and gastric adenocarcinoma susceptibility in the northwest chinese population," International Journal of Molecular Sciences, vol. 16, no. 5, pp. 11648-11658, 2015.

[30] L. J. Engle, C. L. Simpson, and J. E. Landers, "Using highthroughput SNP technologies to study cancer," Oncogene, vol. 25, no. 11, pp. 1594-1601, 2006.

[31] B. A. Salisbury, M. Pungliya, J. Y. Choi, R. Jiang, X. J. Sun, and J. Stephens, "SNP and haplotype variation in the human genome," Mutation Research - Fundamental and Molecular Mechanisms of Mutagenesis, vol. 526, no. 1-2, pp. 53-61, 2003.

[32] T. K. Harden and J. Sondek, "Regulation of phospholipase C isozymes by ras superfamily GTPases," Annual Review of Pharmacology and Toxicology, vol. 46, pp. 355-379, 2006.

[33] T. D. Bunney, R. Harris, N. L. Gandarillas et al., "Structural and mechanistic insights into ras association domains of phospholipase C epsilon," Molecular Cell, vol. 21, no. 4, pp. 495-507, 2006.

[34] O. Pagel, S. Loroch, A. Sickmann, and R. P. Zahedi, "Current strategies and findings in clinically relevant post-translational modification-specific proteomics," Expert Review of Proteomics, vol. 12, no. 3, pp. 235-253, 2015.

[35] S. Mocellin, D. Verdi, K. A. Pooley, and D. Nitti, "Genetic variation and gastric cancer risk: A field synopsis and metaanalysis," Gut, vol. 64, no. 8, pp. 1209-1219, 2015.

[36] H. B. El-Serag, A. C. Mason, N. Petersen, and C. R. Key, "Epidemiological differences between adenocarcinoma of the oesophagus and adenocarcinoma of the gastric cardia in the USA," Gut, vol. 50, no. 3, pp. 368-372, 2002.

[37] J. Powell and C. C. McConkey, "The rising trend in oesophageal adenocarcinoma and gastric cardia," European Journal of Cancer Prevention, vol. 1, no. 3, pp. 265-269, 1992.

[38] L. Wang, X. Bi, X. Song et al., "A sequence variant in the phospholipase $\mathrm{C}$ epsilon $\mathrm{C} 2$ domain is associated with esophageal carcinoma and esophagitis," Molecular Carcinogenesis, vol. 52, no. S1, pp. 80-86, 2013.

[39] S. C. Sorli, T. D. Bunney, P. H. Sugden, H. F. Paterson, and M. Katan, "Signaling properties and expression in normal and tumor tissues of two phospholipase C epsilon splice variants," Oncogene, vol. 24, no. 1, pp. 90-100, 2005. 


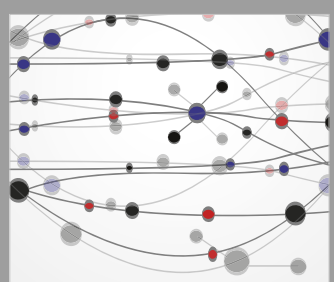

The Scientific World Journal
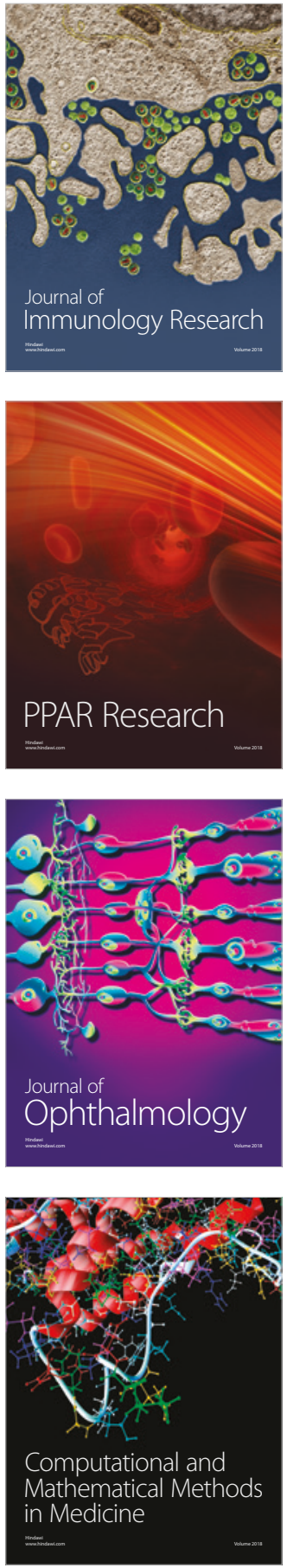

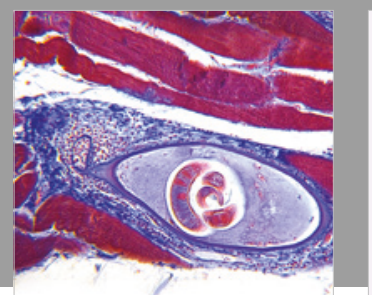

Gastroenterology Research and Practice

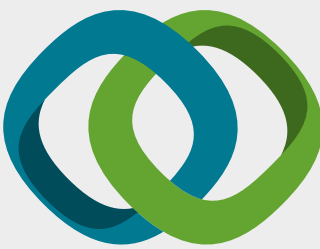

\section{Hindawi}

Submit your manuscripts at

www.hindawi.com
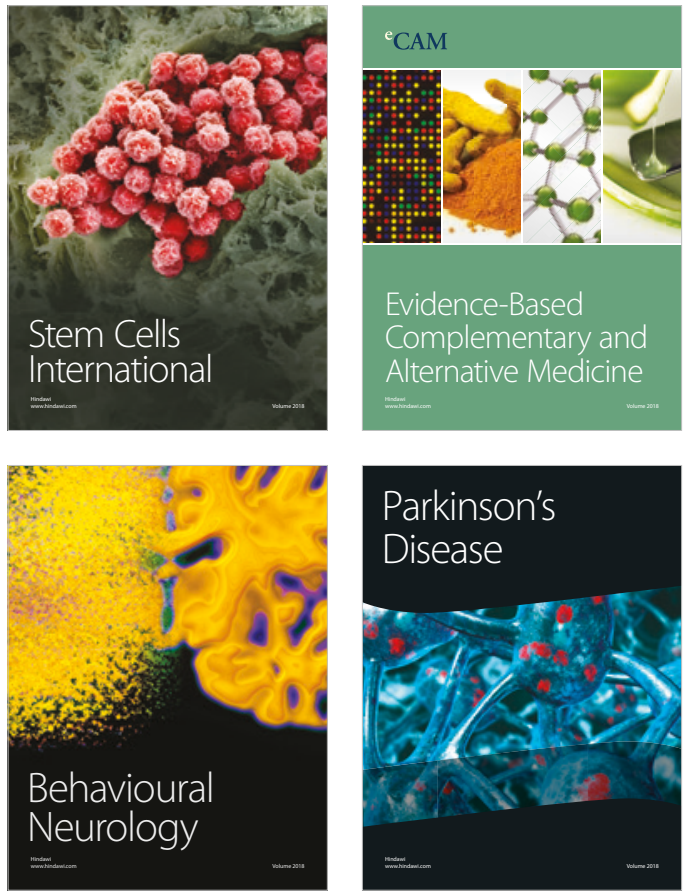

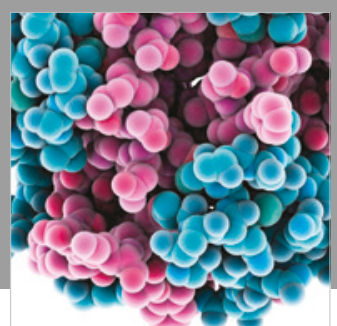

ournal of

Diabetes Research

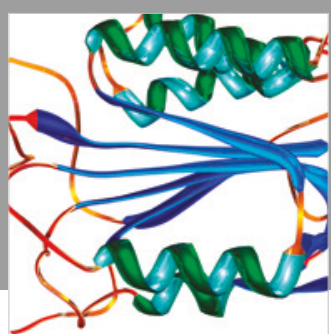

Disease Markers
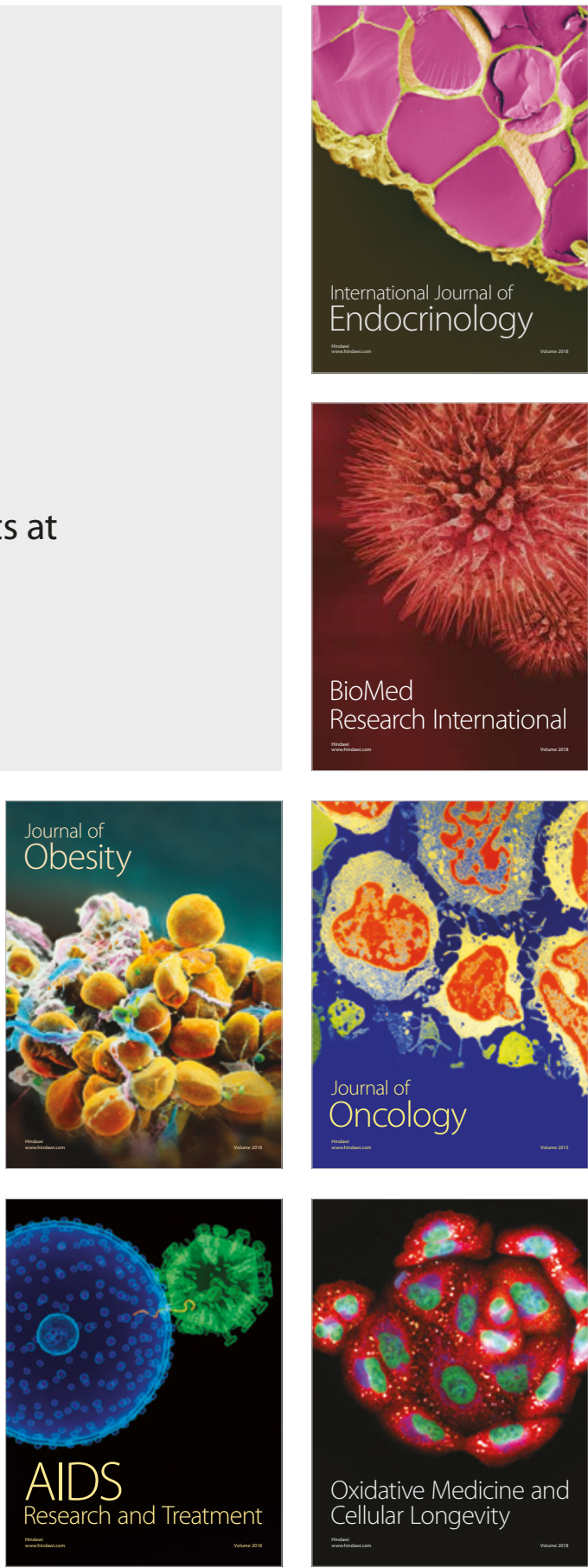